\title{
Dynamic Change of Gut Microbiota in the Male Bee of Bombus terrestris (Hymenoptera: Apidae)
}

\author{
Kai Li ${ }^{1, \dagger}$, Liuhao Wang ${ }^{1,2, \dagger}$, Zhengyi Zhang ${ }^{1}$, Yulong Guo ${ }^{1}$, Jun Guo ${ }^{3}$, Yanping Chen ${ }^{4}$, \\ Daohua Zhuang ${ }^{5}$ \& Jilian $\mathrm{Li}^{1}$
}

${ }^{1}$ Key Laboratory of Pollinating Insect Biology of the Ministry of Agriculture, Institute of Apicultural Research, Chinese Academy of Agricultural Science, Beijing, China

${ }^{2}$ College of Resources and Environmental Sciences, Henan Institute of Science and Technology, Xinxiang, Henan, China

${ }^{3}$ Faculty of Life Science and Technology, Kunming University of Science and Technology, Kunming, Yunnan, China

4 Bee Research Laboratory, United States Department of Agriculture, Agricultural Research Service (USDA-ARS), Beltsville, Maryland, USA

${ }^{5}$ iCarbonX (Shenzhen) Co., Ltd., Shenzhen, China

Correspondence: Jilian Li, Key Laboratory of Pollinating Insect Biology of the Ministry of Agriculture, Institute of Apicultural Research, Chinese Academy of Agricultural Science, Beijing, China. E-mail: bumblebeelj1@hotmail.com

Received: March 16, $2021 \quad$ Accepted: June 26, $2021 \quad$ Online Published: August 15, 2021

doi:10.5539/jas.v13n9p163 URL: https://doi.org/10.5539/jas.v13n9p163

$\dagger$ These authors contributed equally to this work.

\begin{abstract}
The gut microbiota plays a key role in the development and health of bumble bees. Male bees are important for the reproductive activity of a colony, yet there are few studies on their gut microbiota. By using qPCR, we found that significant changes in total bacteria and six important bacteria genera from different developmental age points in males. And we compare the gut bacteria of male bees with workers. The results indicate that Gilliamella, Snodgrassella, and Lactobacillus are the dominant gut bacteria in male bees, which is consistent with the previous studies in worker bees, however, there are more total bacteria in male bees. Another gut bacteria genus, Bacillus may be a probiotic bacteria for reproduction in male bees, although the possible function of these bacteria require further study. This research can provide insight into the relationship between the bacterial community and the physiological health and reproductive capacity of male bumble bees.
\end{abstract}

Keywords: Gilliamella, Snodgrassella, Lactobacillus, Bombus terrestris

\section{Introduction}

Bumble bees are important pollinators and play a key role in maintaining ecological balance and plant diversity (Williams et al., 2009). The members of the colony are divided into three different castes, each with specialized duties: a queen, workers, and males. After the queen has founded a colony and her first clutch of workers emerges to help her in providing for the colony, the queen's primary job is to lay eggs. The workers gather food, care for the young, and clean and defend the nest. The males' purpose is to leave to mate with virgin queens from other nests, ensuring future genetic diversity.

Many studies have suggested that there are close relationships between hosts and their gut microbiota. Some gut bacteria can generate a probiotic effect for the host assisting the host to digest food, while some cause the host to produce anti-microbial peptides and defend against pathogens in honeybees (Evans et al., 2004; Mattila et al., 2012) Similarly, there is a relatively simple yet specialized microbiota in bumble bee gut (Koch et al., 2012; Martinson et al., 2011). Studies have shown that Gilliamella, Snodgrassella, and Lactobacillus are the dominant gut bacteria in bumble bee workers, and these bacteria have an important impact on the bumble bee's development and physiology (Evans et al., 2004). In addition, there is a significant difference between the gut 
bacteria in unmated and mated bumble bee queens. Gilliamella, Snodgrassella, and Lactobacillus are the dominant genera in unmated queens; however, Bacillus, Pseudomonas, and Lactococcus are the main gut bacteria in mated queens, The number of 16S rRNA copies of Gilliamella, Snodgrassella, and Lactobacillus increased with age post-eclosion (1-7 days) in Unmated Queens, declined significantly following mating at 7-days across the Mated Queens stage (1-7 days post mating), but rebounded to the peak in Unmated Queens 7-days post eclosion by the time of queen egg laying (post-hibernation Ovipositing Queens). Bacillus, Lactococcus, and Pseudomonas increased from low levels in the Unmated Queens state, returning to Unmated Queens state abundances in post-hibernation Ovipositing Queens. In the previous study, we found significant shifts in the microbial composition across queen states, with specific microbiome signatures being associated with different stages. Unmated and ovipositing queens showed the greatest similarity in community composition, with mated queens being distinct (Wang et al., 2019), however, it is still unknown whether the gut bacteria change in the post-mating queens are associated with bumble bee males. Additionally, Gilliamella, Snodgrassella, and Lactobacillus have been found to assist bumble bees in degrading the pollen wall, absorbing nutrition and protecting the host against parasites (Koch et al., 2012; Kwong et al., 2014; Sanchez et al., 2011). Further research is required on the possible function of Bacillus, Pseudomonas, and Lactococcus in bumble bees.

In contrast to workers bees, few studies have examined microbial communities that are associated with bumble bee males, even though their health and proper function are important to the productivity of their colonies.

Using a targeted qPCR approach, we assessed the abundance in the identified predominant bacteria (including Gilliamella, Snodgrassella, Lactobacillus, Bacillus, Pseudomonas, and Lactococcus) at different ages of unmated and mated male bees, which may help further the understanding of the relationship between male bees and gut bacteria. A better understanding of the gut microbiota composition in different ages of male bees would shed light on the complex interplay between the microbiota and male health and mating. Our study is the first to explore dynamic changes of the gut microbiota across different ages of males, from adult eclosion to mating. It shows dynamic diversity and variation of gut bacterial communities and improves our understanding of possible relationships between the gut microbial communities and different developmental and ages of males.

\section{Materials and Methods}

\subsection{Sample Collection}

Males of Bombus terrestris (Linnaeus) (Hymenoptera: Apidae) were collected from the Institute of Apicultural Research, CAAS, China. The colonies were reared in the dark at a temperature of $27 \pm 1^{\circ} \mathrm{C}$ and relative humidity of $50-60 \%$. Sugar water $(1: 1 \mathrm{v} / \mathrm{v})$ and apricot pollen were provided ad libitum to subsequently produced 100 colonies until males and gynes (new queens) emerged. Bees in two different physiological states were sampled at different time points. In the unmated samples, we collect the $1 \mathrm{~d}, 3 \mathrm{~d}, 5 \mathrm{~d}, 7 \mathrm{~d}, 9 \mathrm{~d}, 11 \mathrm{~d}, 13 \mathrm{~d}, 15 \mathrm{~d}, 17 \mathrm{~d}, 18 \mathrm{~d}$, $19 \mathrm{~d}, 21 \mathrm{~d}, 23 \mathrm{~d}$, and $25 \mathrm{~d}$ after emergence ( $\mathrm{n}=5$, per time point). And the mated samples including $1 \mathrm{~d}, 6 \mathrm{~d}, 11$ $\mathrm{d}, 16 \mathrm{~d}, 21 \mathrm{~d}$, and $26 \mathrm{~d}$ after mated with queen ( $\mathrm{n}=5$, per time point). All collected samples were treated with liquid nitrogen and then stored at $-80^{\circ} \mathrm{C}$ until used.

\subsection{Extraction of the Gut DNA}

The whole gut (including crop, midgut, ileum, and rectum) of bumble bee males were collected at the sterile environment. For each bee, the body surface was disinfected with $70 \%$ and $90 \%$ ethanol solution for $1 \mathrm{~min}$ respectively, then using the double-distilled water to wash it for some times. The abdomen was dissected with the disinfectant scissors and tweezers, the whole digestive tract was removed and transferred into a $1.5 \mathrm{~mL}$ microcentrifuge tube with $100 \mu \mathrm{L}$ double-distilled water and ceramic beads $(0.1 \mathrm{~mm})$ for the DNA extraction. After the gut sample was homogenized in the Tissue Lyser (QIAGEN Hilden, Germany), the genomic DNA was isolated with the Wizard $^{\circledR}$ Genomic DNA Purification Kit (Promega, A1120), following the manufacturer's instructions, $30 \mu \mathrm{L}$ nuclease-free water was used to dissolve the DNA. The concentration and quality of extracted DNA were determined by the Nanodrop 2000 (Thermofisher) and 1\% agarose gel electrophoresis. The DNA was stored at $-20^{\circ} \mathrm{C}$ until used.

\subsection{The Primer Design and PCR Amplification}

The major bacterial genus' 16S rRNA gene sequences were acquired from the GenBank database. The conserved regions of each genus was aligned and analyzed by using the software DNAMAN, then using Primer Premier (version 5.0) to identify and design the unique primer pairs. The universal 16S rRNA primer was used to detect the total bacterial copies for every sample which was from the previous studies (Martinson et al., 2012; Cariveau et al., 2014). The primer sequences of Bacillus, Pseudomonas, and Lactococcus were BacF (GATGCGTAG CCGACCTGAGA) and BacR (GGCGTTGCTCCGTCAGACTT), PseF (CCGTAACTGGTCTGAGAGGATG) 
and PseR (GCATGGCTGGATCAGGCTTT), LactF (GCGATGATACATAGCCGACCTG) and LactR (AGTTA GCCGTCCCTTTCTGGTT) respectively, primers of 16 s, Gilliamella, Snodgrassella, and Lactobacillus were from the previous study (Xu et al., 2014; Li et al., 2012). In order to ensure the specificity of these primer pairs, the PCR amplification was performed in a $20 \mu \mathrm{L}$ mixture system, which consisting of SYBR ${ }^{\mathbb{B}}$ Premix Ex Taq II (Tli RNaseH Plus) $(2 \times)(10 \mu \mathrm{L})$, the forward primer $(10 \mu \mathrm{M})(0.8 \mu \mathrm{L})$, the reverse primer $(10 \mu \mathrm{M})(0.8 \mu \mathrm{L})$, DNA sample $(1 \mu \mathrm{L})$ and the double-distilled water $(7.4 \mu \mathrm{L})$. And the PCR reaction process was pre-denaturation at $95{ }^{\circ} \mathrm{C}$ for $30 \mathrm{~s}$, then 40 cycles of $95^{\circ} \mathrm{C}, 5 \mathrm{~s}$ for denaturation and $60{ }^{\circ} \mathrm{C}, 30 \mathrm{~s}$ for annealing. The specificity of the amplified fragments was checked by melt curve, and the product sizes were determined by $1 \%$ agarose gel electrophoresis.

\subsection{Absolute Quantification PCR (qPCR)}

Single-band PCR products were purified using the EasyPure PCR Purification Kit and inserted into the T vector by the use of the pEASY-T1 Simple Cloning Kit. The recombinant plasmid DNA was transformed into competent cells. After the mixtures were smeared uniformly on Luria broth (LB) agar plates, they were cultured at $37{ }^{\circ} \mathrm{C}$ overnight, then the positive bacterial clones were selected and continued to culture with liquid LB. The recombinant plasmid DNA was isolated by the AxyPrep Plasmid DNA Mini Kit (Axygen, APMNP 50) according to the manufacturer's instruction. The plasmid concentration was measured by spectrophotometry (Nanodrop 2000, Thermofisher) and the quality was visualized via $1 \%$ agarose gel electrophoresis. The recombinant plasmid DNA was stored at $-80{ }^{\circ} \mathrm{C}$ for future use.

According to the formula described by Dhanasekaran et al. (2010) at the basis of the concentration of recombinant plasmids, the original copy numbers of the recombinant plasmid DNA were calculated and then 10 -fold serially diluted to obtain different concentration for constructing the standard curve.

Absolute quantitative PCR was performed with samples and the serially diluted plasmid DNA simultaneously, the PCR reaction mixture and thermocycler conditions were the same as described above. The samples DNA were diluted 10-fold before use and each of them were run in triplicate. The bacterial actual copy numbers in samples were calculated with the $\mathrm{Ct}$ value which related to the relevant standard curve (Yu et al., 2005), and the standard curve was built by forming a liner regression between the copy number of diluted standards ( $\mathrm{x}$ axis) and the corresponding $\mathrm{Ct}$ values (y axis). The amplification efficiency of the plasmid template was calculated from the slope of the standard curve according to the following formulas: $\mathrm{E}=10^{(-1 / \mathrm{slope})}-1$ (Thermo Fisher Scientific qPCR Efficiency Calculator).

\subsection{Statistical Analysis}

The SPSS software (version 23) was used to analyze the copy numbers of bacterial genera among different samples. The significant differences of bacterial copy numbers at different time points were performed by One-way ANOVAs and Least Significant Difference tests (LSD).

\section{Results}

\subsection{Copy Number Variations of Differentially Abundant Bacterial Genera in the Different Developmental Stages} of Unmated Males

The total bacterial copies and the single bacterial genera of Gilliamella, Snodgrassella Lactobacillus, Bacillus, Pesudomonas, and Lactococcus were detected in unmated males over a period of 1-25 days (Figure 1). The results show that Gilliamella, Snodgrassella and Lactobacillus are the core bacterial in the gut of male bees. And the mean absolute number $( \pm$ s.d.) of the overall bacterial rRNA genes of each age and six predominant genera were quite low at the first day post-eclosion. The copies of total bacteria gradually increased and peaked at the ninth day $\left(1.01 \times 10^{10} \pm 3.53 \times 10^{9}\right)$, after that they began to decline and persisted at a relatively low level during the remaining stages (Figure 1). The change in abundance of Gilliamella and Snodgrassella is similar $(\mathrm{r}=0.833$, $\mathrm{p}=0.0001)$, both began to increase gradually and peaked at the seventh day $\left(3.38 \times 10^{9} \pm 5.64 \times 10^{8}, 5.31 \times\right.$ $\left.10^{9} \pm 7.02 \times 10^{8}\right)$, which is a significant difference to other time points $(\mathrm{p}=0.04)$. Levels then gradually diminished until the fifteenth day $\left(5.35 \times 10^{8} \pm 1.92 \times 10^{8}, 9.91 \times 10^{8} \pm 2.66 \times 10^{7}\right)$, after then they maintained a relatively high level. The change in quantities of Lactobacillus and Lactococcus were similar $(\mathrm{r}=0.949, \mathrm{p}=$ $0.0001)$ with the highest concentration at the third day $\left(1.13 \times 10^{9} \pm 1.52 \times 10^{8}, 1.87 \times 10^{5} \pm 5.65 \times 10^{4}\right)$, which is significantly different to other stages $(\mathrm{p}<0.03)$, the copy numbers remained at a relatively low level at other stages (Figure 1). The highest abundance of Bacillus was at the nineteenth day $\left(3.09 \times 10^{8} \pm 7.65 \times 10^{7}\right)$, which is significantly different to other stages $(\mathrm{p}=0.04)$. The Pseudomonas bacteria was present in greatest amounts at the seventeenth day $\left(2.31 \times 10^{6} \pm 2.39 \times 10^{5}\right.$; Figure 1$)$. 

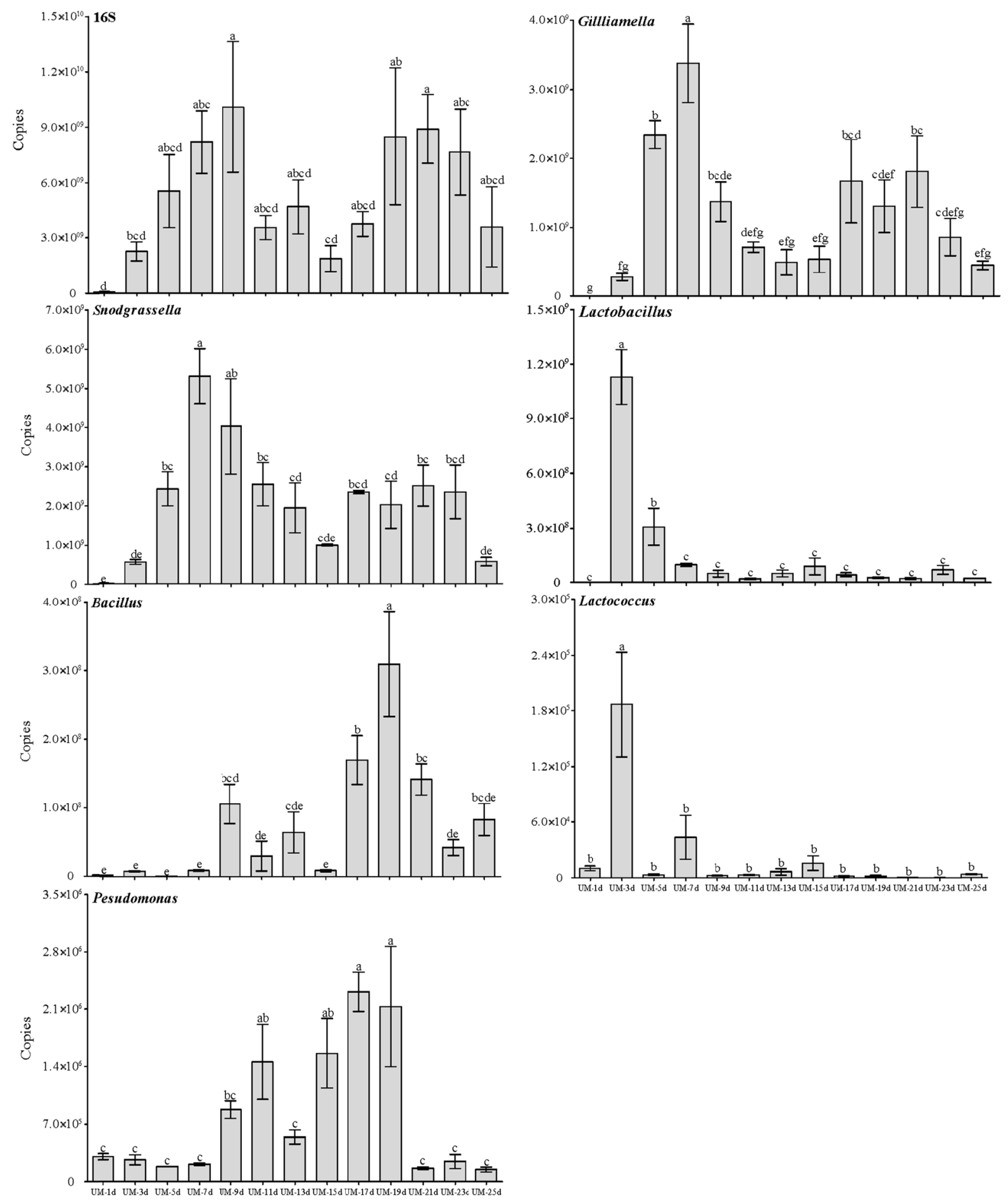

Figure 1. Dynamic changes of gut bacteria (Gilliamella, Snodgrassella, Lactobacillus, Bacillus, Pesudomonas and Lactococcus) in the different time points of unmated male bees. The x-axis are days after eclosure

\subsection{Copy Number Variation of Differentially Abundant Bacterial Genera Compare in the Different Developmental Stages of Mated and Unmated}

In this study, the male bee first mated with the queen at the twelfth day after eclosion. Male bees were detected at the thirteenth day when they emerged from the pupa, and act as the first day of the mated male. The results showed that there was no significant different for the total bacterial copies among the different male stages (Figure 1). However, the Gilliamella and Snodgrassella genera had the highest copy numbers at the first day of mating $\left(3.06 \times 10^{9} \pm 1.02 \times 10^{9}, 8.54 \times 10^{9} \pm 2.94 \times 10^{9}\right)$, which was a significant difference to unmated $(\mathrm{p}=0.039$; 
Figure 2). However, the genera of Lactobacillus $\left(2.19 \times 10^{8} \pm 4.73 \times 10^{7}\right)$ and Pseudomonas $\left(6.70 \times 10^{7} \pm 9.75 \times\right.$ $\left.10^{5}\right)$ had the highest copy numbers at the sixth day for mated males, which was a significant difference to unmated $(p=0.005 ; p=0.001)$; Although the genera of Lactococcus was higher than unmated on 6 days, there was no significant difference $\left(\mathrm{p}=0.094\right.$; mated: $1.23 \times 10^{4} \pm 4.83 \times 10^{3}$; unmated: $1.75 \times 10^{3} \pm 8.78 \times 10^{2}$; Figure 2). Bacillus had the highest copy numbers at the eleventh $\left(9.15 \times 10^{7} \pm 5.39 \times 10^{6}\right)$ and sixteenth days $(8.64 \times$ $\left.10^{7} \pm 1.01 \times 10^{7}\right)$, and there was significant differences to unmated $(\mathrm{p}=0.11 ; \mathrm{p}=0.005$; Figure 2$)$.
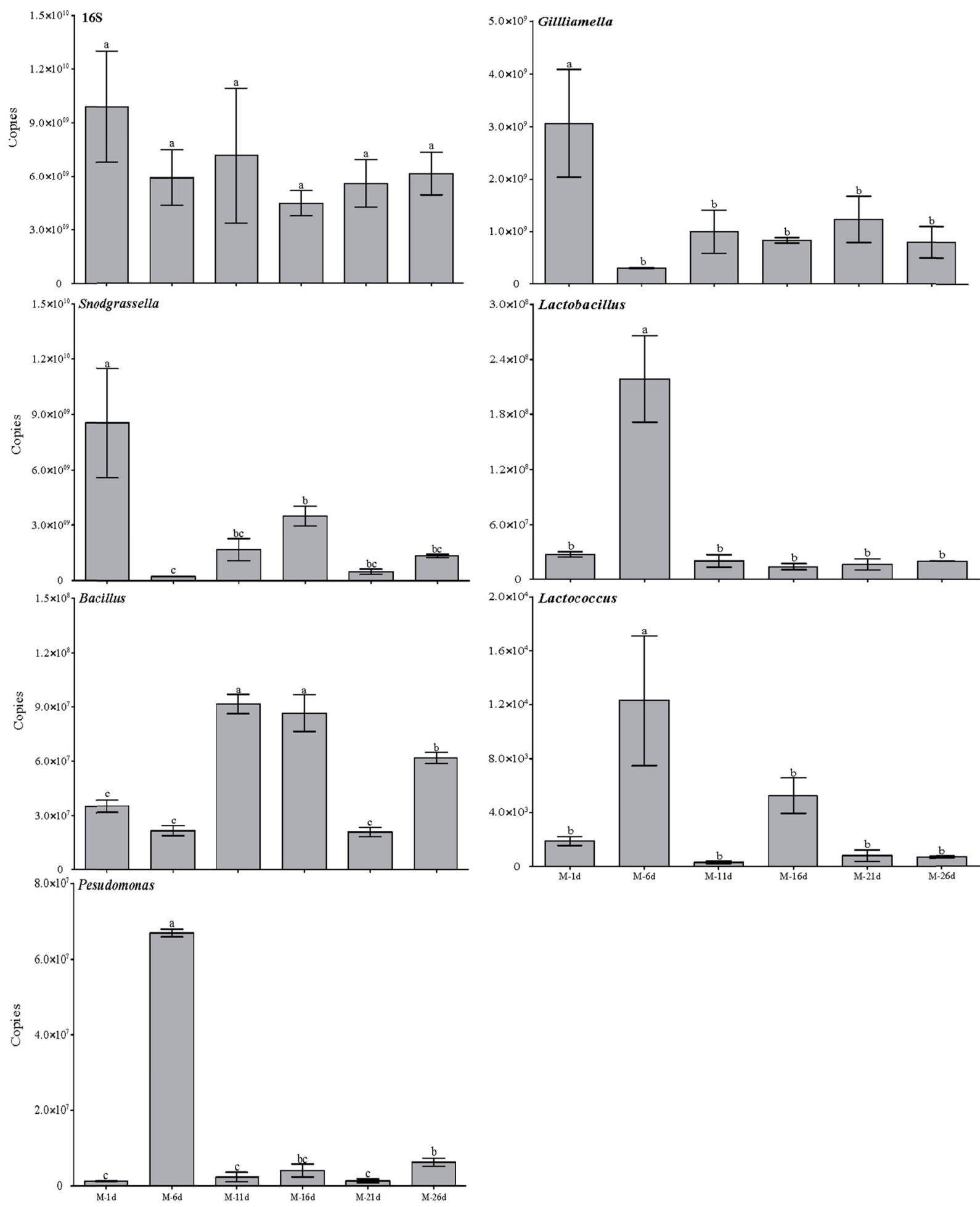

Figure 2. Comparison of gut bacteria (Gilliamella, Snodgrassella, Lactobacillus, Bacillus, Pesudomonas and Lactococcus) in different mating states. The $\mathrm{x}$-axis are days after eclosure 


\section{Discussion}

Previous study had suggested that there has a close relationship between gut microorganisms and their host, the gut microbiota has important impacts on the development and physiology of the host (Yoshiyama et al., 2009; Sabaté et al., 2009; Audisio et al., 2011; Hoy et al., 2003; Knight et al., 2003). However, the host can also influence the abundance and composition of gut microorganisms (Koch et al., 2012; Li et al., 2012; Biagi et al., 2010). In this study, the dynamic change of the total bacteria and each bacterial genus Gilliamella, Snodgrassella, Lactobacillus, Bacillus Lactococcus, and Pseudomonas was identified at different developmental age points of unmated and mated males. The results showed there were different dynamic changes in gut bacteria at different developmental age points, it might be explained that different genus of gut bacteria have different functions in their host at different age points of development. Many studies have found that gut symbionts potentially affect reproductive behaviors in insects. For example, in Drosophila melanogaster, commensal bacteria play a role in mating preferences (Sharon et al., 2011; Engel et al., 2012), and the alteration of female microbiota counteracts a default male outbreeding strategy by inhibiting female sexual signaling (Heys et al., 2018). We estimated the Bacillus decreased post-mating males from high levels in the unmated males. In contrast with our previous study, Bacillus increased post-mating from low levels in the unmated queens (Wang et al., 2019). The differences are intriguing; however, further work is required to clarify if the microbiota influences male bumble bee mating behavior and chemical communication required for copulation-such as male sex pheromone production.

Many studies have demonstrated that the bacterial genus of Gilliamella, Snodgrassella, and Lactobacillus play a key role in the health of worker bees (Yoshiyama et al., 2009; Sabaté et al., 2009; Audisio et al., 2011; Hoy et al., 2003). In previous study, the bacterial genus of Bacillus, Lactococcus, and Pseudomonas shows an obvious increase in the mated bumble bee queens (Wang et al., 2019). Likewise, the genus of Gilliamella and Snodgrassella are also the major gut bacteria in male bees, which is consistent with the previous study on worker bees (Kwong et al., 2014; Xu et al., 2014; Powell et al., 2014). The content of Lactobacillus and Bacillus is relatively low when compared with Gilliamella and Snodgrassella; the metabolic pathway shows that Lactobacillus can transform various carbohydrates into lactic acid (Sanchez et al., 2011; Kleerebezem et al., 2009), and the function is closely associated with the larvae-feeding process, which is done by the workers (Guo et al., 2015). Therefore, levels are higher in the guts of workers than in male bees (Koch et al., 2012). In addition, there are fewer copies of Pseudomonas and Lactococcus, indicating that perhaps they are not the main gut bacteria in male bees.

After emerging from the pupa to the point of sexual maturity, male bees rely on bee bread for nutrition. Gut bacteria abundance is initially low; this gradually increases with the host's development. This result is consistent with the study on worker bees (Martinson et al., 2012; Guo et al., 2015). The core gut bacteria are colonized in the male through the host's contact with its nestmates, hive materials, and consumption of bee bread. During early adulthood, male bees are focused on feeding in order to obtain enough energy. The genomics study of Gilliamella apicola found that it can assist the host in degrading pollen and obtaining adequate nutrition, and Snodgrassella and Lactobacillus can protect the host against pathogens (Kwong et al., 2014; Forsgren et al., 2010), Therefore, they present in high abundance in the guts of male bees, which maybe in order to improve the development and health of their host. Bacillus has relatively high copies from the ninth day after eclosion, and the individual also reaches sexual maturity at this Time (Tasei et al., 1998). Zhang revealed that Bacillus can increase the activity and quality of sperm in male mice, which suggests that gut bacteria may be associated with the host's reproductive success. Additionally, Bacillus can also enhance the host's immunity and protect against pathogens (Zhang et al., 2009; Shao et al., 2018; Aly et al., 2008).

Most bumble bee species only mate with one queen in their lifetime (including Bombus terrestris) (Zhou et al., 2016), and they do not play a role in the colony after mating. This study reveals that the abundance of gut bacteria is maintained at relatively low levels (except for Bacillus) after mating; this can be influenced by the physiological status and the roles of the host.

The Bacillus began to appear in large numbers at 9 days (Figure 1). Studies have shown that Bacillus have certain immunity and anti-microbiotal effects on the host organism (Aly et al., 2008). On the day 6, the Pesudomonas was significantly more than unmatted, and kept consistent with the body of the queen. The Pesudomonas began to increase at the day 9 of emergence. The content of Pesudomonas increased significantly after mating, and the content of Pesudomonas was increased. The increase in the content may be related to a substance that appears in the mating after mating, and Bacillus is significantly more pronounced in the gut after mating than in the unmated queen, but which factor is now specific still uncertain. Males have the function of automatically regulating the microbiota (Guo et al., 2015). 
This study suggests that Gilliamella, Snodgrassella, and Lactobacillus are also the core gut bacteria in male bumble bees, as is the case for worker bees. Yet, Bacillus is also abundant in the bee gut, possibly because it is another probiotic bacterium for the host. Meanwhile, the possible function of these gut bacteria in the growth and development of the host is an area that requires more study.

\section{References}

Aly, S. M., Ahmed, A. G., Ghareeb, A. A., \& Mohamed, M. F. (2008). Studies on bacillus subtilis and lactobacillus acidophilus, as potential probiotics, on the immune response and resistance of tilapia nilotica (Oreochromis niloticus) to challenge infections. Fish. Shellfish. Immun., 25, 128-136. https://doi.org/ 10.1016/j.fsi.2008.03.013

Audisio, M. C., María, J. T., Daniela, C. S., Ibarguren, C., \& María, C. A. (2011). Properties of different lactic acid bacteria isolated from Apis mellifera L. bee-gut. Microbiol. Res., 166, 1-13. https://doi.org/ 10.1016/j.micres.2010.01.003

Biagi, E., Nylund, L., Candela, M., Ostan, R., Bucci, L., Pini, E., ... De Vos, W. (2010). Through ageing, and beyond: Gut microbiota and inflammatory status in seniors and centenarians. PLoS ONE, 5, e10667. https://doi.org/10.1371/annotation/df45912f-d15c-44ab-8312-e7ec0607604d

Cariveau, D. P., Powell, E. J., Koch, H., Winfree, R., \& Moran, N. A. (2014). Variation in gut microbial communities and its association with pathogen infection in wild bumble bees (Bombus). ISME J., 8 , 2369-2379. https://doi.org/10.1038/ismej.2014.68

Dhanasekaranab, S., Mark, D. T., Kenneth, J., \& Trials Study Group. (2010). Comparison of different standards for real-time PCR-based absolute quantification. Journal of Immunological Methods, 354(31), 34-39. https://doi.org/10.1016/j.jim.2010.01.004

Engel, P., Martinson, V. G., \& Moran, N. A. (2012). Functional diversity within the simple gut microbiota of the honey bee. P. Natl. Acad. Sci. USA, 109, 11002-11007. https://doi.org/10.1073/pnas.1202970109

Evans, J. D., \& Lopez, D. L. (2004). Microbiotal probiotics induce an immune response in the honey bee (Hymenoptera: Apidae). J. Econ. Entomol., 97, 752-756. https://doi.org/10.1093/jee/97.3.752

Forsgren, E., Olofsson, T. C., Váasquez, A., \& Fries, I. (2010). Novel lactic acid bacteria inhibiting Paenibacillus larvae in honey bee larvae. Apidologie, 41, 99-108. https://doi.org/10.1051/apido/2009065

Guo, J., Wu, J., Chen, Y. P., Evans, J. D., Dai, R., Luo, W. H., \& Li, J. L. (2015). Characterization of gut bacteria at different developmental stages of Asian honey bees, Apis cerana. J. Invertebr. Pathol., 127, 110-114. https://doi.org/10.1016/j.jip.2015.03.010

Heys, C., Anne, L., Hervé, C., Price, T. A. R., Prescott, M., Ingleby, F., \& Lewis, Z. (2018). Evidence that the microbiota counteracts male outbreeding strategy by inhibiting sexual signaling in females. Front. Ecol. Evol., 6, 29. https://doi.org/10.3389/fevo.2018.00029

Hoy, M. A., Jeyaprakash, A., Alvarez, J. M., \& Allsopp, M. H. (2003). Wolbachia is present in Apis mellifera capensis, A. m. scutellata, and their hybrid in southern Africa. Apidologie, 34, 53-60. https://doi.org/ 10.1051/apido:2002048

Kleerebezem, M., \& Vaughan, E. E. (2009). Probiotic and gut lactobacilli and bifidobacteria: molecular approaches to study diversity and activity. Annu. Rev. Microbiol., 63, 269-290. https://doi.org/10.1146/ annurev.micro.091208.073341

Knight, D. J. W., \& Girling, K. J. (2003). Gut flora in health and disease. The Lancet, 361, 1831. https://doi.org/ 10.1016/S0140-6736(03)13438-1

Koch, H., Cisarovsky, G., \& Schmid-Hempel, P. (2012). Ecological effects on gut bacterial communities in wild bumblebee colonies. J. Anim. Ecol., 81(6), 1202-1210. https://doi.org/10.1111/j.1365-2656.2012.02004.x

Kwong, W. K., Engel, P., Koch, H., \& Moran, N. A. (2014). Genomics and host specialization of honey bee and bumble bee gut symbionts. P. Natl. Acad. Sci. USA, 111, 11509-11514. https://doi.org/10.1073/pnas.14058 38111

Li, J. L., Qin, H., Wu, J., Sadd, B. M., Wang, X., Evans, J. D., .. Chen, Y. P. (2012). The prevalence of parasites and pathogens in Asian honeybees Apis cerana in China. PLoS ONE, 7, e47955. https://doi.org/ 10.1371/journal.pone.0047955 
Martinson, V. G., Danforth, B. N., Minckley, R. L., Rueppell, O., Tingek, S., \& Moran, N. A. (2011). A simple and distinctive microbiota associated with honey bees and bumble bees. Mol. Ecol., 20, 619-628. https://doi.org/10.1111/j.1365-294X.2010.04959.x

Martinson, V. G., Moy, J., \& Moran, N. A. (2012). Establishment of characteristic gut bacteria during development of the honey bee worker. Appl. Environ. Micro., 78, 2830-2840. https://doi.org/10.1128/ AEM.07810-11

Mattila, H. R., Daniela, R., Walker-Sperling, V. E., Guus, R., Newton, I. L. G., \& Amdam, G. V. (2012). Characterization of the active microbiotas associated with honey bees reveals healthier and broader communities when colonies are genetically diverse. PLoS ONE, 7, e32962. https://doi.org/10.1371/ journal.pone.0032962

Powell, J. E., Martinson, V. G., Urban-Mead, K., \& Moran, N. A. (2014). Routes of acquisition of the gut microbiota of the honey bee Apis mellifera. Appl. Environ. Micro., 80, 7378-87. https://doi.org/10.1128/ AEM.01861-14

Sabaté, D. C., Carrillo, L., \& Audisio, M. C. (2009). Inhibition of Paenibacillus larvae and Ascosphaera apis by Bacillus subtilis isolated from honeybee gut and honey samples. Res. Microbiol., 160, 193-199. https://doi.org/10.1016/j.resmic.2009.03.002

Sanchez, B., Gonzalez-Tejedo, C., Ruas-Madiedo, P., Urdaci, M. C., \& Margolles, A. (2011). Lactobacillus plantarum extracellular chitin-binding protein and its role in the interaction between chitin, caco-2 cells, and mucin. Appl. Environ. Micro., 77, 1123-1126. https://doi.org/10.1128/AEM.02080-10

Shao, L., Li, J., Le, L., Wu, K. N., Du, H. M., \& Wang, L. (2018). Effect of Bacillus subtilis and Rhodopseudomonas palustris on immune function for Carassius auratus and study on bacteriostatic activity of their metabolites. Heilongjiang Animal Science and Veterinary Medicine, 20, 176-178.

Sharon, G., Segal, D., Ringo, J. M., Hefetz, A., Zilber-Rosenberg, I., \& Rosenberg, E. (2011). Commensal bacteria play a role in mating preference of Drosophila melanogaster. P. Natl. Acad. Sci. USA, 107, 20051-20056. https://doi.org/10.1073/pnas.1009906107

Tasei, J. N., Moinard, C., Moreau, L., Himpens, B., \& Guyonnaud, S. (1998). Relationship between aging, mating and sperm production in captive Bombus terrestris. J. Apicult. Res., 37, 107-113. https://doi.org/ 10.1080/00218839.1998.11100962

Wang, L. H., Wu, J., Li, K., Sadd, B. M., Guo, Y. L., Zhuang, D. H., ... Li, J. L. (2019). Dynamic changes of gut microbial communities of bumble bee queens through important life stages. mSystems, 4(6), e00631-19. https://doi.org/10.1128/mSystems.00631-19

Williams, P. H., \& Osborne, J. L. (2009). Bumblebee vulnerability and conservation world-wide. Apidologie, 40, 367-387. https://doi.org/10.1051/apido/2009025

$\mathrm{Xu}$, L. L., Wu, J., Guo, J., \& Li, J. L. (2014). Dynamic variation of symbionts in bumblebees during hosts growth and development. Scientia Agricultura Sinica, 47, 2030-2037.

Yoshiyama, M., \& Kimura, K. (2009). Bacteria in the gut of Japanese honeybee, Apis cerana japonica, and their antagonistic effect against Paenibacillus larvae, the causal agent of American foulbrood. J. Invertebr. Pathol., 102, 91-96. https://doi.org/10.1016/j.jip.2009.07.005

Yu, Y., Lee, C., Kim, J., \& Hwang, S. (2005). Group-specific primer and probe sets to detect methanogenic communities using quantitative real-time polymerase chain reaction. Biotechnol. Bioeng., 89, 670-679. https://doi.org/10.1002/bit.20347

Zhang, S. Q. (2009). Effect of Bacillus Pab02 preparationon reproductive function in mice. Sichuan Agricultural University, China.

Zhou, Z. Y. (2016). Comparison of biological characteristics of four Chinese bumble bee species. Chinese Academy of Agricultural Sciences, China.

\section{Copyrights}

Copyright for this article is retained by the author(s), with first publication rights granted to the journal.

This is an open-access article distributed under the terms and conditions of the Creative Commons Attribution license (http://creativecommons.org/licenses/by/4.0/). 